\title{
COLD PLASMA: THE WAY TO IMPROVE THE REPEATABILITY OF SALD ICP-MS ANALYSIS
}

\author{
Marek Stiborek ${ }^{1,2}$, Jan Preisler ${ }^{* 1,2}$, Masoud ShekargoftaR ${ }^{3}$, VIKTOR KanickÝ ${ }^{1,2}$, AND \\ JAKUB KELAR ${ }^{3}$ \\ ${ }^{1}$ Department of Chemistry, Masaryk University, Kamenice 753/5, Brno, 625 00, CZECH REPUBLIC \\ ${ }^{2}$ CEITEC - Central European Institute of Technology, Masaryk University, Kamenice 753/5, Brno, 625 00, \\ CZECH REPUBLIC \\ ${ }^{3}$ R\&D Centre for Low-Cost Plasma and Nanotechnology Surface Modifications, Department of Physical \\ Electronics, Masaryk University, Kotlářská 2, Brno, 611 37, CZECH REPUBLIC
}

\begin{abstract}
This work deals with the preparation of model biological microsamples for Substrate-Assisted Laser Desorption Inductively Coupled Plasma Mass Spectrometry (SALD ICP-MS). This technique provides the direct and fast analysis of liquid samples deposited onto polyethylene terephthalate (PET) plates (substrates) in the form of dried droplets with minimal sample preparation and submicrolitre sample volume requirements. Furthermore, SALD ICP-MS allows for the direct analysis of samples in organic solvents, which cannot be directly nebulised to ICP-MS. These benefits are, however, balanced out by the low degree of repeatability of the assay, which is typically about $30 \%$. One of the approaches to increase the repeatability is a modification of the substrate surface by Diffuse Coplanar Surface Barrier Discharge (DCSBD) with the addition of an internal standard to the sample. Using this approach, a relative standard deviation (RSD) of less than $10 \%$ for model biological samples can be achieved. The only limitation of this technique is the occurrence of the so-called "aging effect", i.e. the gradual return of the modified physicochemical surface properties to their original state.
\end{abstract}

Keywords: SALD ICP-MS, Plasma Treatment, DCSBD, Microsample, Internal Standard

\section{Introduction}

The determination of elements in biological samples is often complicated by complex matrices, the presence of organic solvents, and trace amounts of the elements of interest. For this reason, it is necessary to select a technique that bridges the obstacles mentioned above. Such a technique is Substrate-Assisted Laser Desorption Inductively Coupled Plasma Mass Spectrometry (SALD ICP-MS), which is capable of analysing liquid samples of submicrolitre volumes in the form of dried droplets (stains) on a polyethylene terephthalate (PET) substrate [1-3]. The greatest difficulty of this technique is the low repeatability of the assay. This is caused mainly by the poorly reproducible deposition of droplets that consist of submicrolitre volumes and the inhomogeneity of the stains produced in terms of the area and thickness of the layer of solid residue in the stain.

The assay repeatability can be increased by increasing the surface energy of the substrate and using internal standards that can correct the mentioned effects.

One way to increase the surface energy, i.e. wettability of the substrate, is to expose it briefly to cold plasma,

\footnotetext{
*Correspondence: preisler@chemi.muni.cz
}

specifically Diffuse Coplanar Surface Barrier Discharge (DCSBD) $[4,5]$.

The plasma system of the so-called DCSBD is composed of regularly alternating silver electrodes to which high alternate voltages are applied. The electrodes are located in a dielectric structure consisting of dielectric oil and thin ceramic plates. The most common arrangement of electrodes in this device is in the form of two intermeshing combs. Surface discharges are then generated on the surface of the ceramic plate, serving as a dielectric barrier. This electrode arrangement (plasma in contact with only inert and highly resistant ceramics) can consist of a theoretically infinite amount of $20 \times 8 \mathrm{~cm}$ DCSBD units and thus, it is theoretically possible to achieve an infinite plasma area.

The principle of substrate surface treatment is based on the excitement and ionization of the species by a high voltage discharge and subsequent introduction of the resulting species onto the substrate surface in the form of radicals, ions and excited molecules.

A disadvantage of this technique over other physicochemical modifications is the gradual decrease in surface tension compared to its original value prior to the modification, or the so-called "aging effect" [6]; this period is in the order of several months. Factors influencing the qual- 
Table 1: Ablation parameter settings monitored in addition to the other isotopes of interest.

\begin{tabular}{l|l}
\hline Isotope integration time & $0.1 \mathrm{~s}$ \\
\hline Flow rate of the He carrier gas & $1.01 \cdot \mathrm{min}^{-1}$ \\
\hline Flow rate of the Ar auxiliary gas & $0.61 \cdot \mathrm{min}^{-1}$ \\
\hline Flow rate of the Ar plasma gas & $151 \cdot \mathrm{min}^{-1}$ \\
\hline Laser pulse repetition rate & $10 \mathrm{~Hz}$ \\
\hline Laser-beam waist & $250 \mu \mathrm{m}$ \\
\hline Laser fluence & $\sim 0.75 \mathrm{~J} \cdot \mathrm{cm}^{-2}$ \\
\hline
\end{tabular}

ity of the modification are the voltage and frequency of the electromagnetic field, the composition of the gaseous atmosphere, the exposure time and the geometries of the sample and electrodes.

\section{Experimental}

In the first experiment, a DCSBD device (RPS400Roplass plasma system 400 W, Roplass, Czech Republic) was used to increase the surface energy of the PET substrate (Bayer MaterialScience, Germany). The substrate surface was modified at $300 \mathrm{~W}$ for one, five and ten seconds. Subsequently, $1 \mu \mathrm{l}$ of $0.5 \mathrm{mM}$ trisodium citrate (Sigma-Aldrich, USA) solution was deposited onto ten replicates of the PET substrate as a model biological sample. The contact angles were measured by the instrument (see System E, Advex Instruments, Czech Republic) using a technique called "sitting droplet on a solid foundation". The device consists of a charge-coupled device (CCD) camera placed on a sliding table in front of which a substrate plate is positioned. Using a micropipette, a $1 \mu \mathrm{l}$ droplet of the sample is deposited onto the substrate and the See System software calculates the contact angle based on the interpolation of the height and width of the arc that describes the shape of the drop. The size of the contact angle was evaluated immediately after modification of the substrate surface as well as after one, seven and 31 days.

Based on the previous experiment, where the aging of the modified surface was monitored by varying the contact angle and size of the stain, depending on the dropping time that elapsed from the surface modification, a complementary experiment was performed. $1 \mathrm{~mL}$ of trisodium citrate sample was mixed with cadmium and indium standards (aqueous calibration solutions, certified reference materials (CRM), Analytika, Czech Republic) so that the resulting solution contained $400 \mathrm{ng} \cdot \mathrm{l}^{-1}$ of these standards. Five replicates of the $200 \mathrm{nl}$ droplet sample solutions prepared were immediately applied using a micropipette to the surface of the modified PET substrate as well as one, three and six days after the modification.

The Cd and In standards, chosen because of their similar atomic masses and first ionization energies, were used

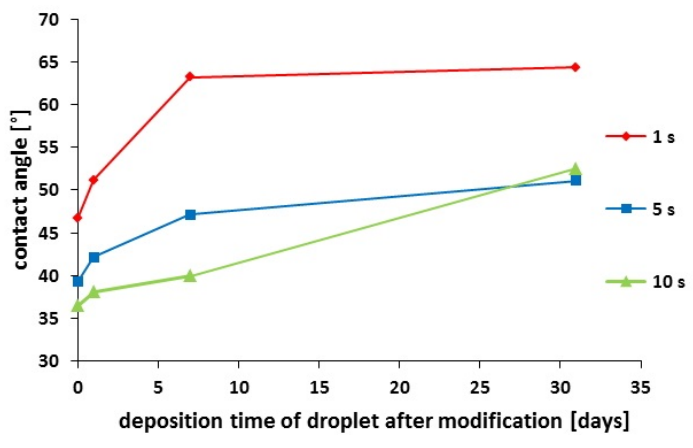

Figure 1: Dependence of the contact angle of droplets of $0.5 \mathrm{mM}$ trisodium citrate solution on the PET surface modified by DCSBD after one, five and ten seconds; droplets were deposited immediately as well as one, seven and 31 days after the modification.

to correct the intensity of the monitored signals. The purpose of this experiment was to demonstrate whether aging of the substrate affects the intensities of the signals. At the same time, the size of the stains was measured. Ideally, the application conditions should be identical to the experiment where aging was observed by measuring contact angles. However, this was not possible due to different sample-volume requirements for the given measuring technique. $1 \mu \mathrm{l}$ was required to measure contact angles and $0.2 \mu \mathrm{l}$ for SALD ICP-MS.

The plate with samples was then inserted into the ablation cell (UP213 model, New Wave Research, Inc., USA) equipped with a 213-nm pulsed Nd:YAG laser, 3D positioning system and built-in CCD camera for visual control of the ablated samples. The dry aerosol created during ablation was analysed by the ICP mass spectrometer (Agilent 7500ce ICP-MS, Agilent, USA). In order to determine the beginning and end of the ablation process, the $13 \mathrm{C}$ isotope was isotope was monitored in addition to the other isotopes of interest.

For the ablation of the stains, a "zig-zag" ablation trajectory was selected with a $170 \mu \mathrm{m}$ ablation line and a $160 \mu \mathrm{m} \cdot \mathrm{s}^{-1}$ scanning speed. The remaining basic ablation parameters are shown in Table 1.

\section{Results and discussion}

First, the contact angle formed by $1 \mu$ d droplets of 0.5 $\mathrm{mM}$ trisodium citrate onto a PET substrate surface was evaluated immediately after modification of the substrate surface as well as after one, seven and 31 days. Ten replicates of the substrate surface were modified for one, five and ten seconds.

Fig. 1 shows that the droplets of $0.5 \mathrm{mM}$ trisodium citrate solution that were applied to the substrate surface of PET modified by DCSBD for one second exhibited the greatest contact angle, hence the least wettability; i.e., a longer duration of DCSBD modification causes a higher degree of wettability. It is also evident from Fig. 1, how- 
Table 2: Integrated SALD ICP-MS signals of ${ }^{111} \mathrm{Cd}$ and ${ }^{115}$ In from stains of $0.5 \mathrm{mM}$ trisodium citrate deposited at different times after PET surface modification.

\begin{tabular}{c|cccc|c}
\hline $\begin{array}{c}\text { Deposition } \\
\text { time [days] }\end{array}$ & & ${ }^{111} \mathrm{Cd}$ & ${ }^{115}$ In & $\begin{array}{c}{ }^{115} \text { In / } \\
{ }^{111} \mathrm{Cd}\end{array}$ & $\begin{array}{c}\text { Stain } \\
\text { size [mm] }\end{array}$ \\
\hline \multirow{2}{*}{0} & $\bar{X}$ [CPS]* & 0.44 & 11 & 25 & $1.2 \pm 0.1$ \\
& RSD [\%] & 14 & 10 & 7.5 & \\
\hline \multirow{2}{*}{1} & $\bar{X}$ [CPS]* & 1.4 & 31 & 22 & $1.6 \pm 0.2$ \\
& RSD [\%] & 34 & 38 & 7.2 & \\
\hline \multirow{2}{*}{3} & $\bar{X}$ [CPS]* & 2.2 & 48 & 23 & $1.4 \pm 0.2$ \\
& RSD [\%] & 32 & 29 & 12 & \\
\hline \multirow{2}{*}{6} & $\bar{X}$ [CPS]* & 2.8 & 58 & 22 & $1.2 \pm 0.3$ \\
& RSD [\%] & 34 & 24 & 19 & \\
\hline
\end{tabular}

${ }^{*} \mathrm{CPS} \cdot 10^{6}$

ever, that the surfaces with the longest DCSBD modification times exhibited the fastest tendency to return to their original states in the long term.

In the following experiments, the aging effect of the substrate surface modified by DCSBD on the intensity of SALD ICP-MS signals was monitored. Five replicates of $200 \mathrm{nl}$ droplets containing $0.5 \mathrm{mM}$ trisodium citrate solution and $400 \mathrm{ng} \cdot \mathrm{l}^{-1}$ of cadmium and indium standards were immediately deposited onto the modified PET surface as well as one, three and six days after modification.

From Table 2, it is apparent that the stain sizes, which should be theoretically dependent on the wettability rate and the aging effect of the modified substrate surface, do not affect the repeatability of the assay for the given samples. Furthermore, it is evident that the immediate average integrated intensity of the ${ }^{111} \mathrm{Cd}$ and ${ }^{115} \mathrm{In}$ signals from the stain ablation of the $0.5 \mathrm{mM}$ trisodium citrate sample as well as one, three and six days after modification of the PET surface differed. This finding is contrary to the assumption that these values should be identical, because in all cases the same amount of sample solution was deposited and the spots were practically $100 \%$ ablated by visual inspection. The repeatability of the ablation was low and relative standard deviation (RSD) values exceeded $30 \%$. Such high values of RSDs could be attributed, in particular, to the character of the sample itself, which was crystalline after drying, and the analytes as they were inhomogeneously distributed therein. However, when quantifying the signal intensity ratio of ${ }^{115} \mathrm{In}$ to ${ }^{111} \mathrm{Cd}$, this inhomogeneity could be corrected, as a result the RSD values varied by around $7.5 \%$ for samples deposited immediately and one day after surface modification of the PET.

The samples on the treated surfaces exhibited the highest wettability and, therefore, it was possible to assume that the analytes were homogeneously dispersed in the stain. The ablation records in Figs. $2 \mathrm{~A}$ and $2 \mathrm{~B}$ also show a higher degree of homogeneity of the stains. Samples applied three and six days after modification exhibited higher corrected values of RSDs: $12 \%$ and $19 \%$, re-
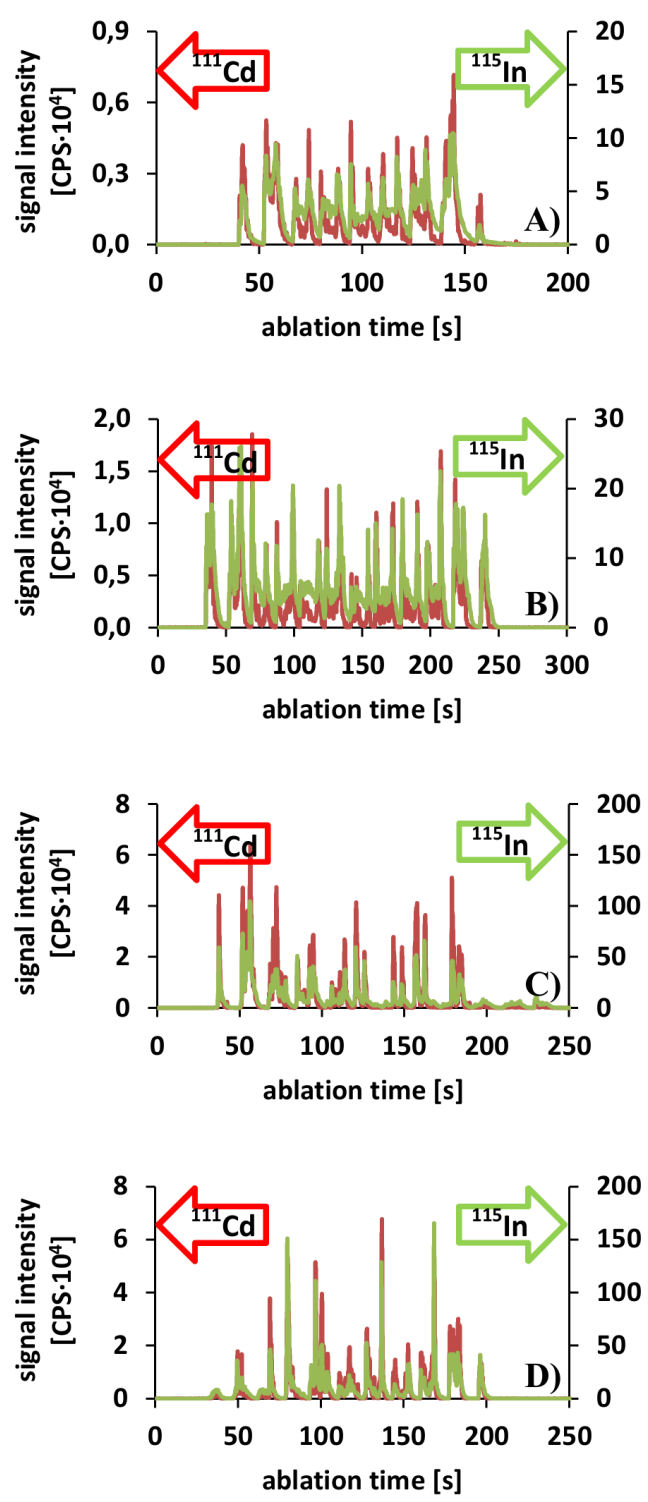

Figure 2: Ablation record of stains of $0.5 \mathrm{mM}$ trisodium citrate deposited A) immediately, as well as B) 1, C) 3 and D) 6 days after PET substrate surface modification.

spectively, mainly due to a higher level of inhomogeneity (Figs. 2C and 2D). Average ${ }^{115} \mathrm{In} /{ }^{111} \mathrm{Cd}$ integrated signal intensity values for the spots applied one, three and six days after surface modification could be used for quantification of the monitored analytes as the values almost coincided. However, the average value of the integrated signal from droplets applied to the substrate immediately after the modification differed. This could be caused by extreme changes in the physical properties of the substrate surface immediately after modification.

\section{Conclusion}

DCSBD technology was chosen for surface modification, i.e. to increase the energy and wettability of the sub- 
strate surface. However, the disadvantage of this technique is the aging of the treated surface, i.e. the return of the modified physicochemical properties of the substrate surface to their original state prior to the modification. The experiments showed that the integrated signal intensities of the selected analytes, ${ }^{111} \mathrm{Cd}$ and ${ }^{115} \mathrm{In}$, present in the desorbed spots of $0.5 \mathrm{mM}$ trisodium citrate solution, that were deposited onto the substrate surface at various time intervals following its modification differed. Also, the repeatability of the analysis of individual samples expressed as RSDs changes with deposition time. RSD values were approximately $30 \%$ for $0.5 \mathrm{mM}$ trisodium citrate samples. When quantifying the ratio of integrated ${ }^{115} \mathrm{In} /{ }^{111} \mathrm{Cd}$ signals, it was found that the values did not differ significantly from each other and could be used in quantitative analysis. Using internal standards, RSD values were reduced to less than $10 \%$ for trisodium citrate samples.

\section{Acknowledgement}

We gratefully acknowledge the financial support of the Czech Science Foundation (15-05387S and 18-16583S) and the Ministry of Education, Youth and Sports of the Czech Republic under the project CEITEC 2020 (LQ1601).

\section{REFERENCES}

[1] Peš, O.; Jungová, P.; Vyhnánek, R.; Vaculovič, T.; Kanick $\imath$, V.; Preisler, J.: Off-Line Coupling of Cap- illary Electrophoresis to Substrate-Assisted Laser Desorption Inductively Coupled Plasma Mass Spectrometry. Anal. Chem. 2008 80(22), 8725-8732 DOI: $10.1021 / \mathrm{ac} 801036 \mathrm{x}$

[2] Navrátilová, J.; Jungová, P.; Vanhara, P.; Preisler, J.; Kanick $\imath$, V.; Šmarda, J.: Copper ions regulate cytotoxicity of disulfiram to myeloid leukemia cells. Int. J. Mol. Med. 2009 24(5), 661-670 DOI: 10.3892/ijmm_00000277

[3] Nehybová, T.; Šmarda, J.; Daniel, L.; Stiborek, M.; Kanick , V.; Spasojevič, I.; Preisler, J.; Damborsk , J.; Beneš, P.: Wedelolactone Acts as Proteasome Inhibitor in Breast Cancer Cells. Int. J. Mol. Sci. 2017 18(4), 729-742 DOI: 10.3390/ijms18040729

[4] Černák, M.; Černáková, L’; Hudec, I.; Kováčik, D.; Zahoranová, A.: Diffuse Coplanar Surface Barrier Discharge and its applications for in-line processing of low-added-value materials. Eur. Phys. J. Appl. Phys. 2009 47(2), 1-6 DOI: 10.1051/epjap/2009131

[5] Štěpánová, V.; Kelar, J.; Galmiz, O.; Zemánek, M.; Slavíček, P.; Buček, A.; Černák, M.: Areal homogeneity verification of plasma generated by diffuse coplanar surface barrier discharge in ambient air at atmospheric pressure. Contrib. Plasm. Physic. 2017 57(4), 182-189 DOI: 10.1002/ctpp.201600093

[6] Prysiazhnyi, V.: Atmospheric Pressure Plasma Treatment and Following Aging Effect of Chromium Surfaces. JSEMAT 2013 3(2), 138-145 DOI: 10.4236/jsemat.2013.32018 